\title{
Collective Synchronization of Undulatory Movement through Contact
}

\author{
Wei Zhou๑, Zhuonan Hao®, and Nick Gravish $\odot^{*}$ \\ Mechanical and Aerospace Engineering, University of California, San Diego, California 92093, USA
}

(Received 12 December 2020; revised 10 May 2021; accepted 23 June 2021; published 7 September 2021)

\begin{abstract}
Many biological systems synchronize their movement through physical interactions. By far, the most well-studied examples concern physical interactions through a fluid: Beating cilia, swimming sperm and worms, and flapping wings all display synchronization behavior through fluid mechanical interactions. However, as the density of a collective increases, individuals may also interact with each other through physical contact. In the field of "active matter" systems, it is well known that inelastic contact between individuals can produce long-range correlations in position, orientation, and velocity. In this work, we demonstrate that contact interactions between undulating robots yield novel phase dynamics such as synchronized motions. We consider undulatory systems in which rhythmic motion emerges from timeindependent oscillators that sense and respond to an undulatory bending angle and speed. In pair experiments, we demonstrate that robot joints will synchronize to in-phase and antiphase oscillations through collisions, and a phase-oscillator model describes the stability of these modes. To understand how contact interactions influence the phase dynamics of larger groups, we perform simulations and experiments of simple three-link undulatory robots that interact only through contact. Collectives synchronize their movements through contact as predicted by the theory, and when the robots can adjust their position in response to contact, we no longer observe antiphase synchronization. Lastly, we demonstrate that synchronization dramatically reduces the interaction forces within confined groups of undulatory robots, indicating significant energetic and safety benefits from group synchronization. The theory and experiments in this study illustrate how contact interactions in undulatory active matter can lead to novel collective motion and synchronization.
\end{abstract}

DOI: 10.1103/PhysRevX.11.031051

\author{
Subject Areas: Biological Physics, \\ Interdisciplinary Physics, \\ Nonlinear Dynamics, Soft Matter
}

\section{INTRODUCTION}

The study of oscillations in biological systems has led to a fundamental understanding of the dynamics of coupled oscillators [1,2]. Biological locomotion typically arises from oscillatory movements, and groups of living systems can exhibit coupled movement oscillations when interacting. For example, recent studies have demonstrated that fluid forces acting between pairs of flagella [3-5], arrays of cilia [6-8], and even flapping wings [9-11] can lead to phase and frequency synchronization of oscillatory body movements. However, many animal and robot groups operate in close proximity where movements may result in collisions, leading to collective jamming [12,13], disorder-to-order transitions in traffic flow [14,15], and

\footnotetext{
*ngravish@ucsd.edu
}

Published by the American Physical Society under the terms of the Creative Commons Attribution 4.0 International license. Further distribution of this work must maintain attribution to the author(s) and the published article's title, journal citation, and DOI. synchronization of oscillatory swimming gaits [16]. In this work, we study the phase dynamics of oscillators that are coupled only through intermittent mechanical contact. We provide experimental and theoretical evidence that inelastic mechanical collisions between independent oscillators produce a rich array of phase dynamics in contact-coupled systems.

Synchronization in biological systems can be observed across all scales-from genetic oscillators within cells $[17,18]$ to collective animal groups within habitats [19-21]. While synchronization is observed across a wide variety of different systems, ultimately it requires two fundamental properties [22]: (1) Perturbations to the phase of each oscillator neither grow nor decay, and (2) oscillator interactions can influence the phase. Many mechanical systems possess both such properties-for example, the original pendulum clocks of Huygens [23] exhibit (1) autonomous oscillations that (2) interact through structural motion. In the context of undulatory locomotion, there are two main archetypes for autonomous oscillations [24]: central pattern generators that provide an adaptive global "clock," and reflexive oscillators that generate spontaneous oscillations through local feedback. Critically, both 
modalities incorporate environmental and proprioceptive feedback. Many abstractions of these circuits exist [25-27], and one common model is the phase oscillator, which oscillates at a constant frequency $\omega$ and can be augmented with sensory feedback.

Collectives that interact through contact have been extensively studied in the soft-matter fields, such as active matter and granular materials. Inert systems that interact through contact, such as granular materials, exhibit novel nonlinear phenomena such as inelastic collapse [28,29], jamming [30], and transitions between fluid and solid states [31]. However, granular materials require external driving forces to stay in motion. In contrast, active matter systems generate spontaneous movement through internal energy reservoirs and external interactions with the group [32]. Studies of self-propelled "dry" active matter have demonstrated that inelastic collisions are responsible for the collective motion and long-range order in dynamical states, such as flocking, jamming, and phase separation (see Ref. [33] for a review). Recent experiments and simulation of undulatory active matter systems such as swimming sperm [34,35] and reciprocating robots [36,37] have demonstrated that contact interactions can lead to novel spatial ordering. However, the explicit ability for these mobile systems to synchronize through contact is unknown. In this paper, we study an active matter system of undulatory robots and demonstrate that inelastic mechanical collisions produce a rich dynamics of collective behavior through contact coupling alone.

As a first example of contact-coupled synchronization, we introduce the Newton's cradle toy (Fig. 1). Newton's cradle is a series of metal balls mounted on wires so that they each undergo pendular motion. When one ball is (a)

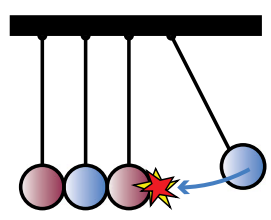

(c)

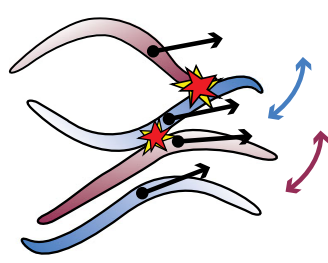

(b)

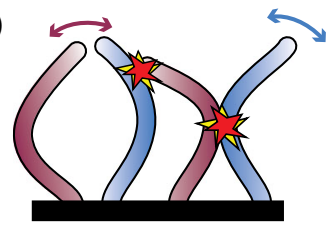

(d)

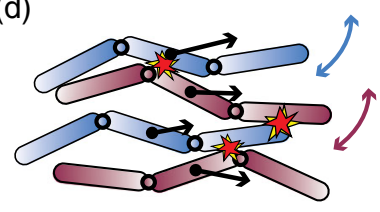

FIG. 1. Examples of oscillators that interact through contact. (a) The balls of the Newton's cradle toy collide and synchronize to in-phase oscillations. (b) Arrays of flapping cilia in close proximity can be driven to synchrony through contact [38]. (c) The undulatory gaits of swimming worms (C. elegans) synchronize through contact interactions [16]. (d) Simple three-link "Purcell-swimmer" robots similarly synchronize their gaits through contact, as demonstrated in this work. allowed to fall under pendular motion and collide with the group, energy is transferred through collisions (with some energy loss), and the ball on the other end will rotate upwards. A less-appreciated aspect of this process is that, as time evolves, energy is lost due to collisions, and eventually the system settles into a state where all of the pendulums are oscillating in phase and in continuous contact. This is a simple example of a contact-coupled dynamical system in which the pendulum is initially out of phase, but through repeated collisions and energy loss, the system is driven to a synchronous oscillating state.

In active oscillating systems, energy loss through dissipation or collisions can be compensated for by energy input, thus exhibiting limit-cycle oscillations [21]. The oscillatory movements of some biological systems can be considered as limit-cycle oscillators [25,39], prompting our interest in the phase dynamics of active oscillatory systems that interact through contact (Fig. 1). We consider simplified representations of biological systems that move through undulation: Our experimental robots use rotary joints and have rigid links. To allow the robots to evolve in the undulatory phase, we use a simple, autonomous, nonlinear oscillator to drive sinusoidal motions of the robots.

In the following sections, we study how oscillatory systems can achieve synchronization when they interact through contact. In Sec. II, we present a simple theoretical model of contact synchronization, and we analyze the steady-state modes and their stability through a contactto-contact iterated map. In Sec. III, we introduce a simple experiment to examine how two robot joints can synchronize through mechanical contact, and we compare with the theoretical model. To understand how contact interactions may apply to larger groups, we perform simulations of 1D lattices and study their dynamics in Sec. IV. In the next two sections, we perform experiments and simulations on simple three-link robots that interact through collisions. We demonstrate that the in-phase synchronization predicted by our phase model, and observed in our first experiments, is observed in robot groups (Sec. V). To find out why synchronization is beneficial in mobile groups, we measure contact forces between robots and compare between the synchronous and asynchronous states (Sec. VI). When robot joint oscillation is driven through time-dependent sinusoidal control, the contact forces are orders of magnitude larger than when the robots are allowed to synchronize.

\section{MODEL OF SYNCHRONIZATION THROUGH CONTACT}

We begin by studying a simple model of two phase oscillators that represent body-bending elements, or the joints of undulatory robots [Fig. 2(a)]. We consider that undulatory motion is generated according to the phase oscillator equation, $\dot{\phi}=1$. The oscillator phase governs the lateral position of the undulating body element, such that 
$x_{i}=A \cos \left(\phi_{i}\right)$ is the lateral distance from the body's center line, and $\dot{x}_{i}=-A \sin \left(\phi_{i}\right)$ is the lateral speed [Fig. 2(b)]. When two body elements are in close proximity, they will come into contact when the following condition is met: $A \cos \left(\phi_{1}\right)-A \cos \left(\phi_{2}\right)=d$, where $d$ is the separation distance of the central axis of the two agents. We introduce the normalized separation distance, $\tilde{d}=(d / 2 A)$, such that only when $\tilde{d} \leq 1$ will oscillators be able to come into contact.

The contact condition thus becomes

$$
\cos \left(\phi_{2}\right)-\cos \left(\phi_{1}\right)=2 \tilde{d} .
$$

When the oscillator pair collides, each oscillator has a velocity of $\dot{x}_{i}^{-}=-A \sin \left(\phi_{i}^{-}\right)$, where superscripts \pm denote before (-) and after $(+)$ collision variables [Fig. 2(b)]. We model the collision as an inelastic process with coefficient of restitution $r$ such that $\dot{x}_{1}^{+}-\dot{x}_{2}^{+}=-r\left(\dot{x}_{1}^{-}-\dot{x}_{2}^{-}\right)$. Combining the inelastic collision model with conservation of momentum, $\dot{x}_{1}^{+}+\dot{x}_{2}^{+}=\dot{x}_{1}^{-}+\dot{x}_{2}^{-}$, yields the following postcollision velocities (we assume equal masses):

$$
\begin{aligned}
& \dot{x}_{1}^{+}=\frac{1}{2}\left[(1-r) \dot{x}_{1}^{-}+(1+r) \dot{x}_{2}^{-}\right], \\
& \dot{x}_{2}^{+}=\frac{1}{2}\left[(1-r) \dot{x}_{2}^{-}+(1+r) \dot{x}_{1}^{-}\right] .
\end{aligned}
$$

When the oscillators collide, they instantaneously change their phase due to the velocity change [Fig. 2(b)]. The oscillator phase is represented in the phase plane as the clockwise angle from the positive $x$ axis to the instantaneous coordinate $(x, \dot{x})$. Thus, the phases before and after a collision can be represented by the following equation:

$$
\phi_{i}^{ \pm}=\operatorname{atan}\left[-\frac{\dot{x}_{i}^{ \pm}}{x_{i}^{ \pm}}\right] .
$$

The negative sign accounts for the fact that the rotation direction is in the clockwise direction.

We seek to understand the asymptotic behavior of the phase difference, $\Delta=\phi_{2}-\phi_{1}$. In systems with continuous coupling, this often amounts to demonstrating that $\dot{\Delta}=0$ [22]. However, since this system consists of repetitive collision events, the phase difference $\Delta$ is constant in between collisions and changes instantaneously during a collision. Thus, we derive the iterated map that takes the precollision phase difference of the $(n)$ th collision to the precollision phase difference of the $(n+1)$ th collision, $\Delta^{(n+1)}=f\left(\Delta^{(n)}\right)$. We represent the phase difference of the $n$th collision as $\Delta^{(n)}$, where we have dropped the superscript \pm for notational convenience. To derive $f\left(\Delta^{(n)}\right)$, we take the following steps: (1) Starting with an initial phase difference, $\Delta^{(n,-)}$, solve for the phases at collision, $\phi_{1}^{-}$and $\phi_{2}^{-}$, (2) apply the velocity update rule for the inelastic collision, and (3) determine the postcollision phases for the oscillators. Since $\omega$ is the same between each oscillator, the $n$th postcollision phase difference is exactly the same as the $(n+1)$ th precollision phase difference, and thus $\Delta^{(n,+)}=\Delta^{(n+1,-)}$. We have now determined the function that generates $\Delta^{(n+1,-)}$ from $\Delta^{(n,-)}$, and we can drop the \pm superscripts, yielding $\Delta^{(n+1)}=f\left(\Delta^{(n)}\right)$. This process results in the collision-to-collision return map (a)

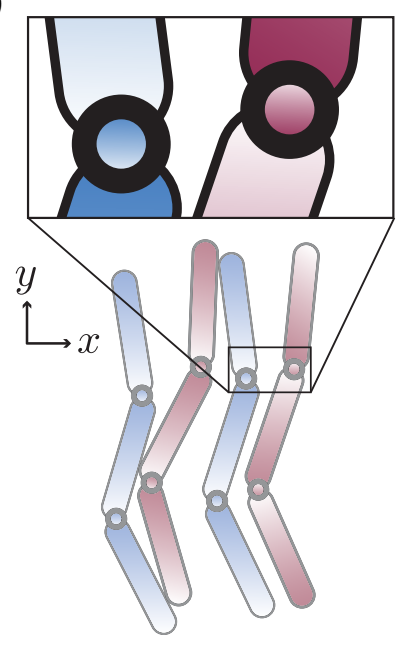

(b)

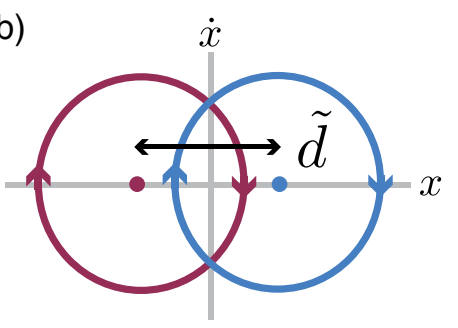

3) Inelastic collision

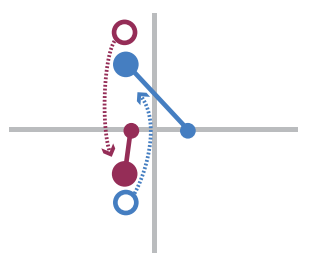

1) Phases before $\mathrm{n}^{\text {th }}$ collision

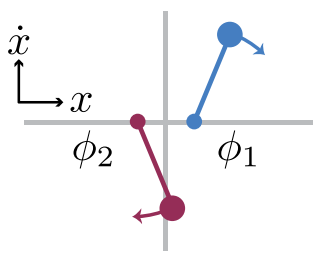

4) Post collision

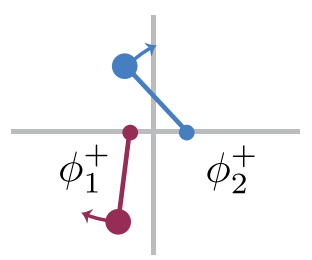

2) Instant before $n^{\text {th }}$ collision

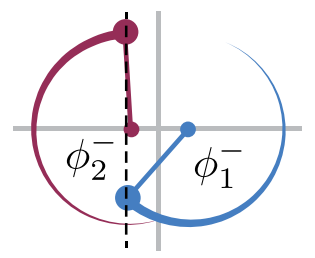

5) Instant before $n+1^{\text {th }}$ collision

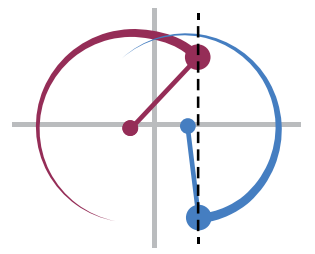

FIG. 2. Phase oscillator model for contact-mediated synchronization of undulatory gaits. (a) Undulatory motion is generated through periodic bending of body elements at joints. (b) We envision that the motion of the body in the lateral direction $(x)$ is governed by a phase oscillator that produces harmonic motion. The evolution of the collision model is shown in steps 1-5. (1) Oscillators are initially at phase difference $\Delta=\phi_{2}-\phi_{1}$. (2) Oscillators will collide when $x_{1}=x_{2}$. (3) During a collision, the velocities are instantaneously updated according to an inelastic collision law, and the phase difference changes. (4) Immediately following collision, the oscillators continue evolving until (5) they collide again, resulting in a new postcollision phase difference. 


$$
\Delta^{(n+1)}=\operatorname{atan}\left[\frac{\sin (\kappa) \cos \left(\frac{\Delta^{(n)}}{2}\right)-r \cos (\kappa) \sin \left(\frac{\Delta^{(n)}}{2}\right)}{\cos (\kappa) \cos \left(\frac{\Delta^{(n)}}{2}\right)-\sin (\kappa) \sin \left(\frac{\Delta^{(n)}}{2}\right)}\right]-\operatorname{atan}\left[\frac{\sin (\kappa) \cos \left(\frac{\Delta^{(n)}}{2}\right)+r \cos (\kappa) \sin \left(\frac{\Delta^{(n)}}{2}\right)}{\cos (\kappa) \cos \left(\frac{\Delta^{(n)}}{2}\right)+\sin (\kappa) \sin \left(\frac{\Delta^{(n)}}{2}\right)}\right],
$$

where we have defined $\kappa=\operatorname{asin}(\tilde{d} \csc (\Delta / 2))$.

The collision-to-collision return map allows us to examine the asymptotic behavior and dynamics of synchronization for contact-coupled oscillators. We first examine the fixed points of the map, $\Delta^{*}=f\left(\Delta^{*}\right)$. The return map for $0<r \leq 1$ exhibits three fixed points as a function of separation. Two of the fixed points exist independently of the coefficient of restitution,

$$
\begin{gathered}
\Delta^{*}=2 \operatorname{asin}(\tilde{d}), \\
\Delta^{*}=\pi,
\end{gathered}
$$

while the third fixed point depends on $r$ and must be solved numerically. We define the first fixed point [Eq. (6)] as the compatibility curve because it defines the maximum phase difference between two oscillators separated by $\tilde{d}$ before they collide [see inset in Fig. 3(a)]. When oscillators are at the compatibility fixed point, they will repeatedly make grazing contact with each other. The compatibility curve actually determines the boundary of an entire set of fixed points for these oscillators since if the phase difference $\left|\Delta^{*}\right|<2 \operatorname{asin}(\tilde{d})$, the oscillators will never contact each other and thus $\Delta$ will never change. The second fixed point [Eq. (7)] is an antiphase oscillation. We show the fixed points in Fig. 3(a) for $r=0.67$, where the lower branch is the compatibility fixed point and the upper branch is the antiphase fixed point.
When the separation distance is zero $(\tilde{d}=0)$, the compatibility fixed point corresponds to perfect in-phase synchronization, $\Delta^{*}=0$, and the return map dramatically simplifies to

$$
\Delta^{(n+1)}=-2 \operatorname{atan}\left(r \tan \left(\frac{1}{2} \Delta^{(n)}\right)\right) .
$$

This equation can be solved recursively to generate the phase difference of the $n$th collision as a function of any initial condition $\left(\Delta^{(0)}\right)$,

$$
\Delta^{(n)}=2 \operatorname{atan}\left((-r)^{n} \tan \left(\frac{1}{2} \Delta^{(0)}\right)\right),
$$

and we clearly see that, for large $n$, the phase difference converges to $\Delta^{*}=0$.

Equation (9) highlights the importance of inelastic collisions in the synchronization process for contactcoupled oscillators. The coefficient of restitution, $r$, governs the rate of convergence to the synchronization fixed point for $\tilde{d}$. The linear stability of fixed points in the collision-to-collision map is determined by the condition $\left|f^{\prime}(0)\right|<1$, where the prime denotes the derivative with respect to $\Delta^{(n)}$. For the $\Delta^{*}=0$ fixed point, the stability is $f^{\prime}(0)=-r$, again highlighting the importance of inelasticity in the synchronization process. Thus, because
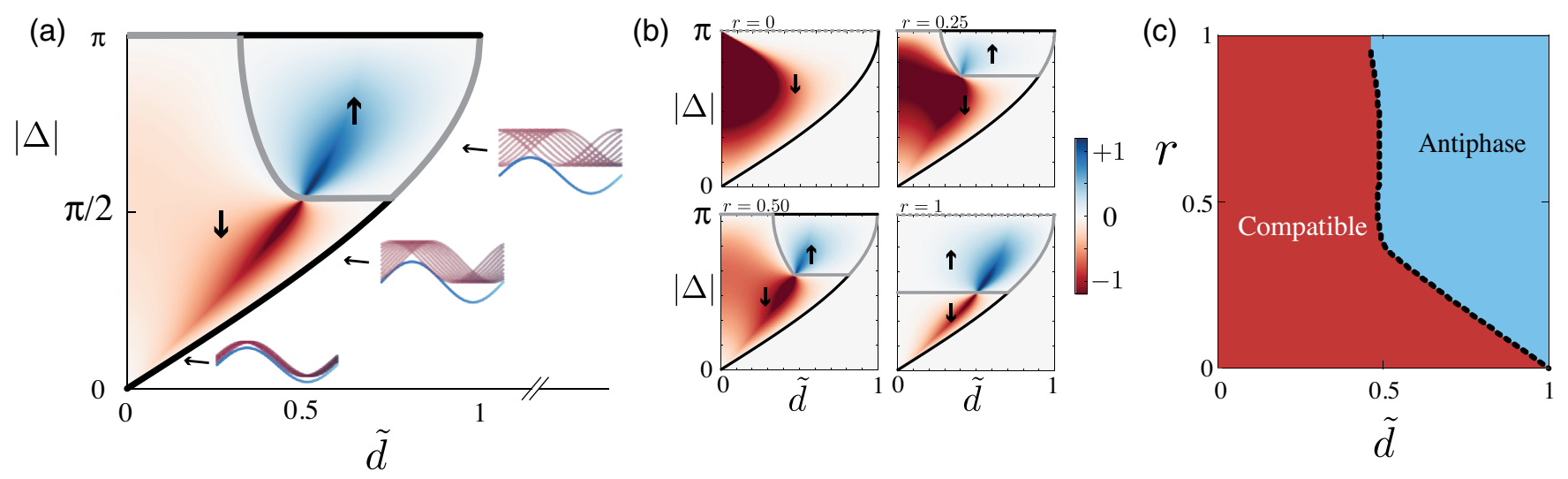

FIG. 3. Evolution of the phase difference captured by the phase oscillator model. (a) For an initial phase difference $\Delta$ and separation distance $\tilde{d}$, a collision will induce a change in $\Delta$. The heatmap shows the collision-to-collision phase difference, $\left|\Delta^{(n+1)}\right|-\left|\Delta^{(n)}\right|$ at each $(\tilde{d}, \Delta)$ location [Eq. (5)]. States in the red region result in a decrease in the absolute phase difference, while states in the blue region increase in phase difference. Black lines are stable fixed points, solid gray lines are unstable, and dashed gray lines are marginally stable. The lower black curve is the compatibility fixed point [Eq. (6)]. The coefficient of restitution is $r=0.67$. The inset shows three different separation distances and the range of "compatible" phase differences that can exist without collision. (b) Collision-to-collision phase change behavior at four different restitution coefficients. The black lines are stable fixed points, the gray lines are unstable fixed points, and dashed gray lines are neutrally stable. (c) Average steady state from random initial phases as a function of separation distance and restitution coefficient. 
inelastic interactions always generate energy loss $(0<r<1)$, the system is guaranteed to reach phase synchronization when $\tilde{d}=0$.

To analyze the time evolution of the system when $0<\tilde{d}<1$, we construct the basins of attraction for the fixed points by calculating the phase change behavior after a single collision event, $g\left(\left|\Delta^{(n)}\right|\right)=\left|\Delta^{(n+1)}\right|-\left|\Delta^{(n)}\right|$. In Figs. 3(a) and 3(b), we plot $g\left(\left|\Delta^{(n)}\right|\right)$ and denote with arrows and color the flow direction of the compatible (down arrow, red) and antiphase (up arrow, blue) basins. We observe that, for each $r$, there is a critical $\tilde{d}_{c}$ below which all initial phase differences are attracted to the compatible state. However, for larger $\tilde{d}$, the antiphase basin causes states that start with large $|\Delta|$ to evolve to antiphase synchronization [blue regions in Figs. 3(a) and 3(b)].

To analyze the behavior of the antiphase fixed point [Eq. (7)], we similarly construct the basin of attraction and linear stability. Since the return map at $\Delta^{*}=\pi$ has a continuous first derivative, we can compute the linear stability of this point. Evaluating the derivative, we find

$$
f^{\prime}(\pi)=-\frac{r\left(d^{2}-1\right)+d^{2}}{r^{2}\left(d^{2}-1\right)-d^{2}},
$$

which yields the critical separation distance, $\tilde{d}_{c}=$ $\sqrt{\left[r(r-1) / r^{2}-r-2\right]}$. When $\tilde{d}>\tilde{d}_{c}$, antiphase oscillations switch from unstable to stable. However, as $r \rightarrow 0$, the range of $\tilde{d}$ where $\left|f^{\prime}(\pi)\right|<1$ becomes vanishingly small as $f^{\prime}(\pi)$ converges to $f^{\prime}(\pi)=1$ for infinitesimal $\tilde{d}$. The overall influence of $r$ and $\tilde{d}$ can be understood by averaging the collision-to-collision phase change across all initial phases, highlighting that, for modest $r \approx 0.5$ and above, the average steady-state behavior is evenly divided between the antiphase and compatible states [Fig. 3(c)].
In this section, we have proven that a simple model of phase oscillators interacting through intermittent inelastic collisions can produce a rich range of dynamical behavior. We observe in-phase synchronization for small separation distances and antiphase synchronization for larger distances. Furthermore, this system admits a continuum of "fixed points" when the phase difference is below the compatibility line, in which case the oscillators are completely uncoupled and do not come into contact. In the next sections, we demonstrate, in experiment and simulation, that the pairwise interactions of contact-coupled oscillators lead to rich collective behaviors.

\section{SYNCHRONIZATION OF ROBOT JOINTS IN EXPERIMENT}

To validate the model introduced in the previous section, we perform experiments with two oscillating motors that interact through collisions [Fig. 4(a)]. Each brushless dc motor (Quanum 5250) represents the joint of a robot and is actuated under closed-loop torque control. Rigid 9-cm-long aluminum links and viscoelastic bumpers are attached to both motors [Fig. 4(a)]. We measure the experimental coefficient of restitution of the system to be $r=0.67$ (Fig. 11 in Ref. [40]). A capacitive encoder attached to the motor shafts provides angular position measurements at a resolution of 8192 counts per revolution, which is $0.044^{\circ}$ (AMT102, CUI Devices). An ODrive brushless dc motor controller (ODrive Robotics) provides closed-loop torque control for both motors individually.

We consider the joint rotation angle and rotational velocity as the position and velocity variables of our phase oscillator, $(x, \dot{x})$. In order to actuate these motors as phase oscillators, we control the motor torque (at a rate of $300 \mathrm{~Hz}$ ) using the following equation: (a)
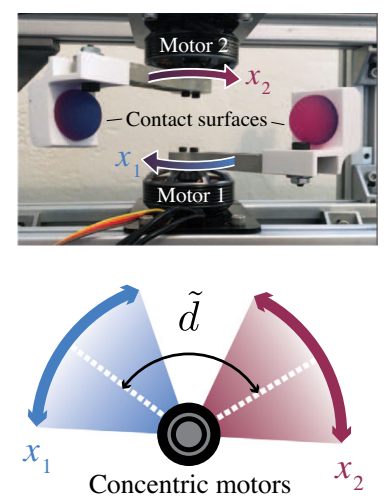

(b)
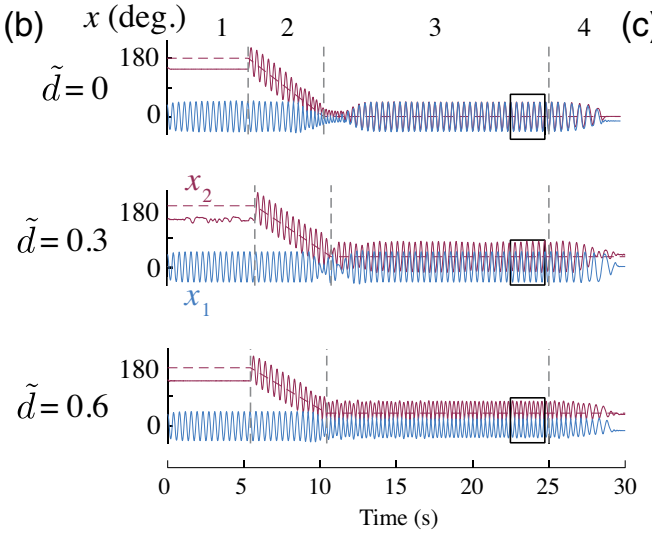

(c)
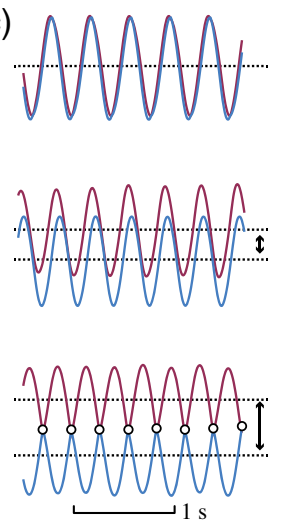
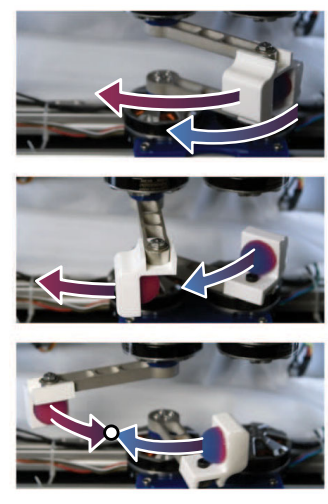

FIG. 4. Experimental validation of synchronization between undulatory robot joints. (a) Two motors mounted on a concentric axis are actuated as phase oscillators. The oscillators interact through inelastic collisions when their rotation angle is equal. (b) Data from three separation distances. Robot joints are initially oscillated out of contact to achieve steady-state behavior (phases 1 and 2 ) and are slowly brought into contact (phase 2) to their final fixed distance $\tilde{d}$ (phase 3), until the experiment is over (phase 4). (c) At low $\tilde{d}$, joints synchronize; at intermediate $\tilde{d}$, joints oscillate with compatible phases and do not come into contact; and at large $\tilde{d}$, joints collide in antiphase synchronization. Images are from Video 1 in Ref. [40]. 


$$
\tau_{i}=-k x_{i}+\left(c-\mu x_{i}^{2}\right) \dot{x}_{i},
$$

where $x_{i}$ is the relative angular displacement from the neutral angle and $i$ refers to the oscillator. We assume the motor internal damping and friction are small, and the system's inertia $(I)$ is the same for both motors, such that $I \ddot{x}_{i}=\tau_{i}$. Note that there is no coupling between the motors in Eq. (11); the only interactions are through inelastic collisions.

The motor actuation in Eq. (11) represents a generic form of the Van der Pol oscillator, which generates sinusoidal oscillations with constant phase speed $(\dot{\phi})$ for weak nonlinearity [21]. Thus, this choice of actuation enables the robot joints to oscillate sinusoidally with constant phase velocity consistent with our phase oscillator model in the previous section. The position and velocity feedback terms in Eq. (11) enable the actuator to instantaneously respond to collision-induced velocity changes, also consistent with our model assumptions. The actuation parameters of Eq. (11) are chosen such that the oscillators have natural frequencies of $\omega_{1}=2.61 \pm 0.04 \mathrm{~Hz}$ and $\omega_{2}=2.63 \pm 0.03 \mathrm{~Hz}$ and amplitudes of $A_{1}=44.4 \pm 0.9$ degrees and $A_{2}=44.3 \pm 1.6$ degrees. For the purposes of analysis and variable definitions, we assume equal amplitudes between the oscillators.

To study the phase dynamics between the two colliding oscillators, we set up steady limit-cycle oscillations with the systems initially separated by a large neutral position, $\tilde{d}=2$. The lower link is allowed to oscillate, and after a random time in the range of 5-7 seconds, the upper link is perturbed to limit-cycle oscillations. This random wait time sets a random initial phase difference between the two oscillators. Once both links are oscillating at a steady state, we slowly move the neutral position of the second oscillator to the prescribed separation $\tilde{d}$ for that experiment. Once the oscillators are at the appropriate $\tilde{d}$, we continue the experiment for 15 seconds until reducing the amplitude and stopping. We measure the oscillator positions and velocities throughout the experiment [Fig. 4(b)] and compute collisional information, including the phase difference before each collision, $\Delta^{(n)}$. In total, we performed 1312 experiments over a range of separation distances where collisions were possible, $\tilde{d} \in[0,1]$, and a control separation distance $\tilde{d}=2$ to rule out any coupling through the structure. In Figs. 4(b) and 4(c) and Video 1 in Supplemental Material [40], we show experiments from three $\tilde{d}$, showing in-phase synchronization $(\tilde{d}=0)$, compatibility $(\tilde{d}=0.3)$, and antiphase synchronization $(\tilde{d}=0.6)$.

We first compare the model predictions and experiment for the $\tilde{d}=0$ return map [Eq. (8)]. In experiment, the oscillators synchronize phases through repeated collision events, eventually reaching a final synchronized state where the oscillators move together in or near contact [Figs. 4(b) and 4(c)]. The experimental collision-to-collision return map shows consistent in-phase synchronization as predicted by Eq. (8) from all initial conditions [Fig. 5(a)]. To rule out the effect of slowly bringing the oscillators together [Phase 2 in Fig. 4(b)], we perform a second set of experiments in which both oscillators begin at $\tilde{d}=0$ and a random initial phase. We observe good agreement in the collision-to-collision return map between both experimental
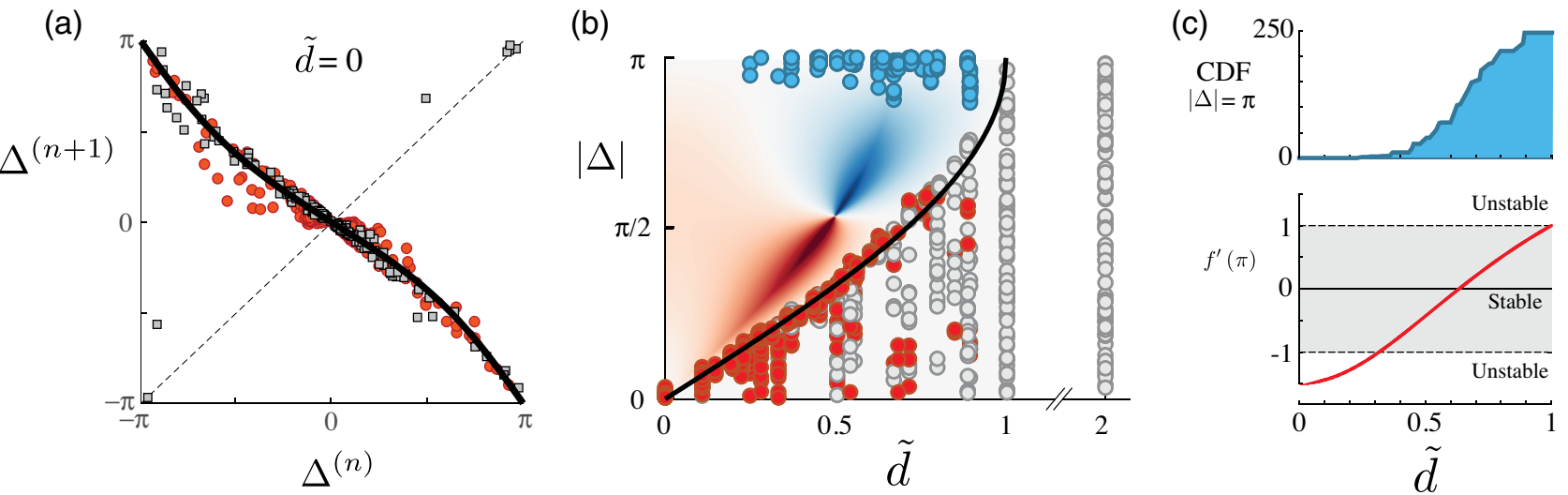

FIG. 5. Experiment results. (a) Collision return map from two separate experimental methods (over 100 experiments for each). Squares are from experiments in which limit cycles slowly move together to $\tilde{d}=0$; circles are experiments in which oscillators are initialized with $\tilde{d}=0$. The black line is return map for $r=0.67$ [Eq. (8)]. (b) Steady-state phase difference $(\Delta)$ versus separation distance $(\tilde{d})$ from 1312 experiments. The black line represents the compatibility curve [Eq. (6)]. Gray and red circles are points that reach a steady-state configuration in which they no longer collide. Red circles start with $|\Delta|$ above the compatibility curve and evolve downwards to the compatible state, while gray circles represent initial conditions below the compatibility line. Blue points are states that evolve to stable antiphase oscillations in which the oscillators repeatedly collide head-on. The heatmap is the model prediction from the collision-to-collision return map for $r=0.67$ [Eq. (5)]. Far right points at $\tilde{d}=2$ are control experiments. (c) Experimental observation of antiphase oscillations coinciding with the onset of antiphase stability in the model. The top is the cumulative distribution of observations of antiphase oscillations versus $\tilde{d}$. The bottom is the stability eigenvalue of antiphase behavior for $r=0.67$ [Eq. (10)]. 
methods. The model and experimental return map exhibit excellent agreement, indicating that the phase-oscillator model is able to capture relevant phase dynamics of this system [Eq. (8); black curve in Fig. 5(a)]. It is important to note here that there are no fitting parameters in the model. Since the phase dynamics are evaluated from collision to collision, we do not need to match frequencies or amplitude between experiment and model. The prediction only requires knowledge of one parameter, the coefficient of restitution $r$, which can easily be measured.

We next compare the steady-state $\Delta$ across the full experimental range of $\tilde{d}$ [Fig. 5(b)]. Comparison of the theoretical compatibility curve [Fig. 5(b), solid line] and the experimental data indicates good agreement between the phase oscillator model and observation. We observe that initial phases that start in the compatible state will continue to stay there [gray circles, Fig. 5(b)], and initial phases that start outside of the compatible state may either evolve to antiphase oscillations or compatibility depending on initial conditions. The red circles in Fig. 5(b) show initial conditions that began above the compatibility line and evolved to the compatible state. Blue circles represent initial conditions that began above the compatibility line and evolved to the antiphase state [Fig. 5(b)].

The antiphase state consists of the two oscillators repeatedly colliding with each other (see Video 1 in Ref. [40]) in a rather violent manner, which leads to broken components on more than one occasion. The antiphase state observed in experiment is found to be remarkably stable and able to resist manual perturbations consistent with the stability calculations in Sec. II. In one experiment, we observe that the two oscillators remain in the antiphase state for over 12 hours until we eventually halt the experiment. The return map allows us to predict when antiphase oscillations become stable [Eq. (10)]. In Fig. 5(c), we compare the cumulative observations of antiphase oscillations and the linear stability calculation [Eq. (10)]. Once again, we find exceptional agreement between the model and experiment: As the antiphase fixed point in our model becomes stable, we begin observing antiphase oscillations in experiment.

\section{COLLECTIVE BEHAVIOR OF MOBILE AND STATIONARY OSCILLATOR GROUPS}

We next seek to understand whether contact interactions among larger groups can yield similar synchronization and phase dynamics as the robot-pair experiments. We consider the lateral dynamics of arrays of mobile cilia and groups of swimming worms as a one-dimensional lattice, where nearest-neighbor collisional interactions occur along the direction of body undulations [Fig. 6(a)]. To simulate the dynamics of mobile and fixed systems, we allow the neutral position of each oscillator to move in response to a collision. Immediately after a collision, we update the neutral positions of the colliding oscillators according to the equation $\delta_{i}=\beta\left(\dot{x}_{j}^{-}-\dot{x}_{i}^{-}\right)$, where $\delta_{i}$ is the neutral position change of the $i$ th oscillator and $\beta$ is the magnitude of collision-induced change. When $\beta=0$, the system base is immobile (as in arrays of cilia) while nonzero $\beta$ allows for oscillators to repel each other through collisions. To confine the oscillator group to a fixed linear distance, we set $\beta=0$ for the left $(i=0)$ and right $(i=N)$ oscillators in the $N$-oscillator lattice. We perform numerical simulations of the one-dimensional oscillator lattice over varied initial neutral positions spanning $\tilde{d}_{i, i+1} \in[0.06,1.2]$. We simulate 50 oscillators initialized at random phases and observe the phase dynamics, collision rate, and neutral position of the group over time.

When the oscillator lattice is initiated in close proximity (small $\tilde{d}_{i, i+1}$ ), the oscillators rapidly converge to a compatible state through collisions [Fig. 6(b)] in both the immobile and mobile cases. Collisions between oscillators initially occur due to the random incompatible phases, and over time, the collision rate decreases, ultimately halting after a time $t_{\text {relax }}$ for small $\tilde{d}_{i, i+1}<0.5$. Once all oscillators are in the compatible state, they will stay there indefinitely unless perturbed.

To quantitatively compare the oscillator lattice results with the theoretical model and experiments from the previous section, we measure the nearest-neighbor phase difference $\left|\Delta_{i, i+1}\right|$ and the nearest-neighbor separation distance of the neutral position, $\tilde{d}_{i, i+1}$. Examining the relationship between the phase difference and spatial separation reveals a fundamental difference between mobile and immobile systems [Fig. 6(c)]. Immobile oscillator lattices show good agreement between the theoretical predictions and simulation for $\tilde{d}_{i, i+1}<0.5$. However, when the separation distance is large $\left(\tilde{d}_{i, i+1}>0.5\right)$, the collisions never stop and the oscillator groups never entirely reach the compatible state [Fig. 6(d), top]. We measure the collision rate over the last approximately 70 periods of oscillation and observe a sharp rise in nonzero steady-state collisions when $\tilde{d}_{i, i+1}>0.5$ for the immobile system. This is supplemented by the large cluster of points above the compatibility curve in Fig. 6(b) for the immobile case. To characterize this deviation from the model prediction, we calculate the fraction of nearest-neighbor pairs that are above the compatibility curve and plot this compatibility error in Fig. 6(d). The immobile base simulations exhibit a large compatibility error and persistent colliding among the group when $\tilde{d}>0.5$.

The immobile system's deviation from the compatibility curve is easily understood from the pairwise dynamics of oscillators modeled and studied in the previous sections. At larger separation distances, the collision-to-collision phase change causes oscillator pairs to increase in phase difference. This phase repulsion is what leads to the stable antiphase mode in the pair experiment. However, in larger groups, the interior oscillators have a left and a right 


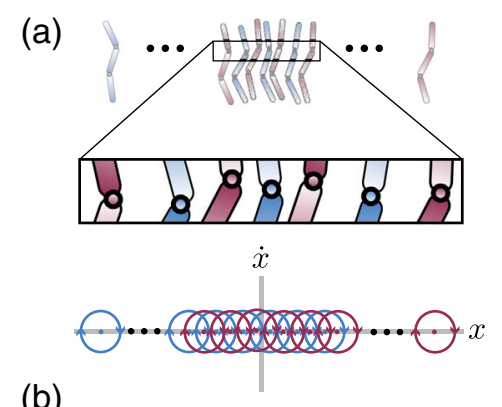

(b)

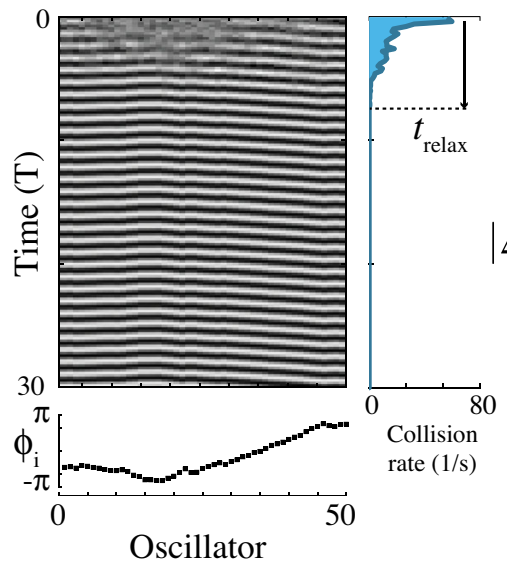

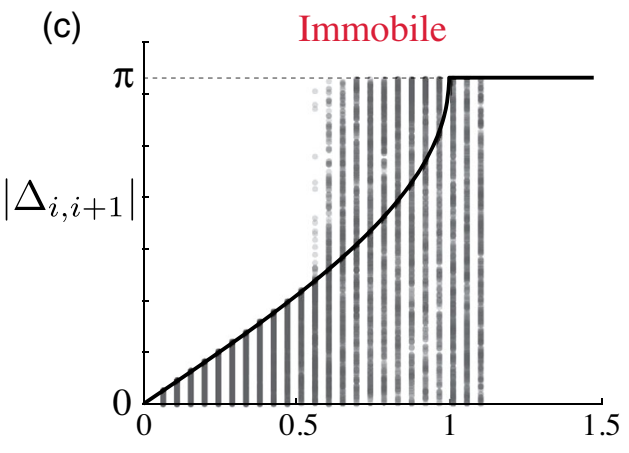

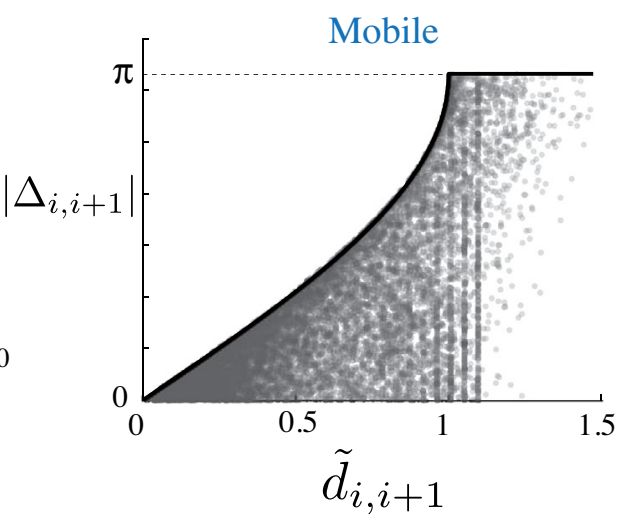

(d)
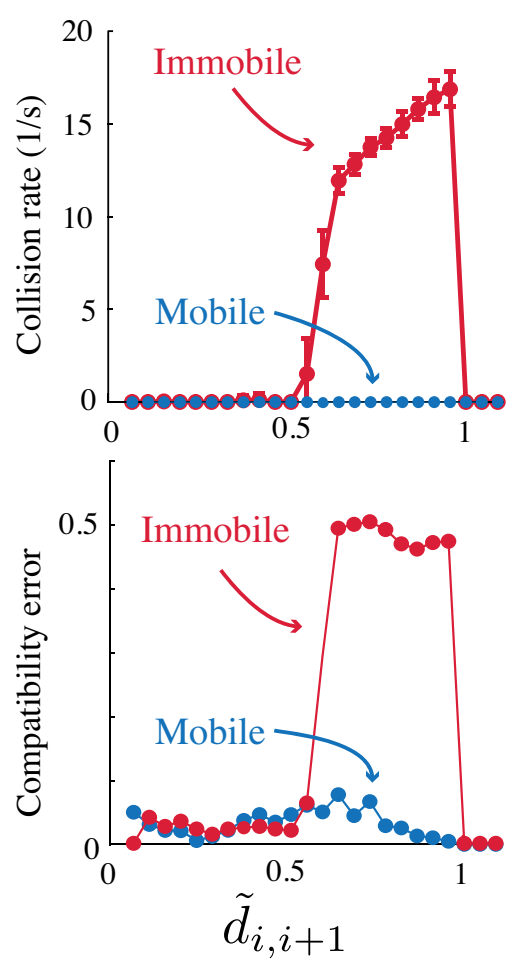

FIG. 6. Phase dynamics for oscillator lattices. (a) Phase dynamics of undulatory robots modeled as a one-dimensional lattice with interactions occurring along the lateral direction. Body undulations between neighboring robots can lead to contact. (b) Spatiotemporal evolution of phase for an oscillator group (50 oscillators, $\tilde{d}=0.14, \beta=0$ ). The bottom plot shows final phases. The right plot shows the rate of collisions and the relaxation time $t_{\text {relax }}$ after which no collisions occur. (c) Final phase difference and separation distance between adjacent oscillators. The top plot shows the phase behavior for oscillators with an immobile base $(\beta=0)$. The bottom plot shows results for mobile oscillators $(\beta=0.1)$ in which the equilibrium position moves as a result of collisions. (d) Collision rate and compatibility error versus $\tilde{d}$. Mobile oscillators always evolve to states with no collisions (top plot) and good agreement with the compatibility equation (bottom plot).

neighbor and thus experience phase repulsion from both of these neighbors which ultimately inhibits the ability to synchronize. These results are in agreement with observations from lattices of locally coupled Kuramoto oscillators in which repulsive phase interactions have been demonstrated to generate asynchronous collective states [41].

In contrast to the immobile system, oscillators that are able to move in response to collisions always relax to the compatible state. The phase and spatial values clustered at or below the compatibility curve [Fig. 6(c)] and exhibit low numbers of collisions and low compatibility error in the steady state [Fig. 6(d)]. The small but nonzero compatibility error for the mobile system is likely due to the assumptions of pure sinusoidal motion in the theory, compared to the slight deviation in sinusoidal behavior that Eq. (11) generates. The deviation from compatibility in the mobile system is still small and clustered on or just above the compatibility line.

The ability for mobile systems to always achieve compatibility can be understood by examining the phase and space dynamics from our theoretical model. In the fixed base system, the only free degree of freedom is $\Delta$, and thus oscillator pairs can only increase or decrease in phase difference (the state evolution in Fig. 3 is only vertical). However, when the base is allowed to move in response to
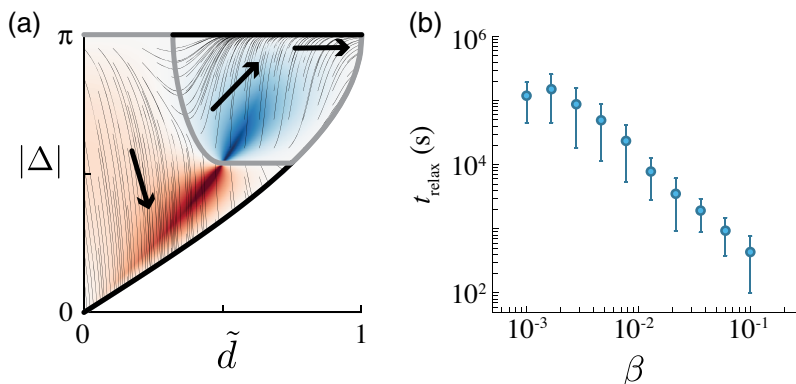

FIG. 7. Collision-induced mobility allowing groups to reach compatibility. (a) Phase and spatial evolution of mobile oscillator groups. Collisions result in an increase in separation distance; thus, the system evolution tends towards larger $\tilde{d}$, and there is no longer a stable antiphase state. (b) System relaxation time versus mobility coefficient $\beta$ for $\tilde{d}=0.78$. As $\beta$ decreases, the relaxation time increases. Here, $\beta=0$ coincides with the immobile simulation, in which case the system evolves to stable antiphase behavior. 
collisions, the oscillator pairs have a second degree of freedom, and the system can evolve through phase change or spatial separation change. Because the spatial change between oscillator pairs is only repulsive, this emerges as a lateral drift towards larger $\tilde{d}$ in the collision-to-collision state evolution [Fig. 7(a)]. Antiphase oscillations are no longer a stable fixed point because the high-impact collisions will drive the oscillators apart until they settle at the point $(\tilde{d}=1, \Delta=\pi)$.

To demonstrate that spatial movement inhibits antiphase oscillation, we examine the long time dynamics of an oscillator lattice initialized at a separation distance that leads to antiphase oscillation in pairs and repetitive collisions in groups $(\tilde{d}=0.78)$. We vary the magnitude of collision-induced spatial change, $\beta$, over 2 orders of magnitude and observe a nearly 3-order-of-magnitude increase in the relaxation time [Fig. 6(b)]. This powerlaw behavior matches previous simulations and intuition from our model: Immobile systems will never relax to collisionless compatibility since $t_{\text {relax }} \rightarrow \infty$ as $\beta \rightarrow 0$. Thus, we see a fundamental difference between mobile and fixedbase systems that undulate and interact through collisions, and these results suggest that mobile robots and organisms will always evolve to compatible, collisionless states through contact.

\section{ROBOTS SYNCHRONIZE GAITS THROUGH COLLISIONS}

Lastly, we examine how groups of mobile undulatory robots synchronize their gaits through contact. We perform both experiments and simulations with simple three-link robots that have two active servomotors (Dynamixel AX12 , Robotis) controlling joint angles $\alpha_{1}, \alpha_{2}$, and three rigid links of length $18.65 \mathrm{~cm}$ [Fig. 8(a)]. Such a three-link system is often referred to as "Purcell's swimmer" and was originally introduced by Purcell as a minimal model of low Reynolds swimming [42]. The three-link robot has been studied extensively in the context of locomotion through fluids $[43,44]$, on frictional surfaces $[45,46]$, and within granular media [47]. In addition, three-link robots have been recently used to study the collective behavior of robot groups that exhibit time-dependent oscillatory motion and push each other through contact $[36,37,48]$.

In experiment, the robot actuators are controlled by continuously sending position commands for the joint angle at a rate of $100 \mathrm{~Hz}$. To actuate the robot joints according to the phase-oscillator model using position-controlled servos, we numerically integrate the oscillator equation used in the motor pair experiments [Eq. (11)] solving for the next joint angle at each time step. Critically, this actuation method requires measuring the instantaneous joint angle and joint (a)

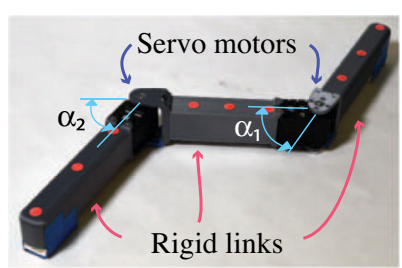

(c)

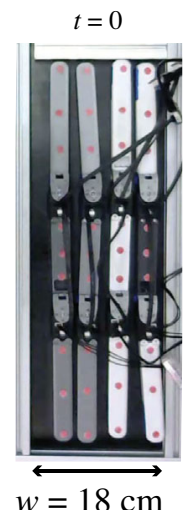

(b)

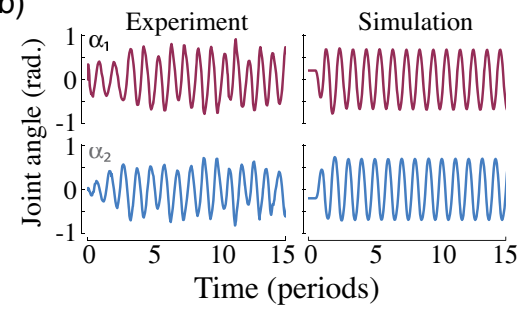

(d)

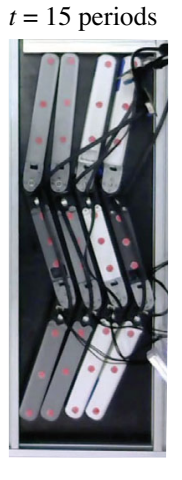

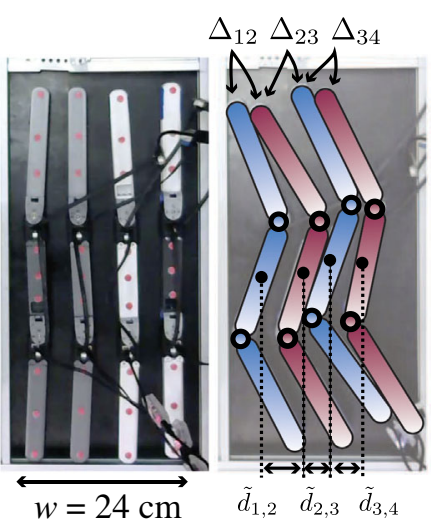

(e)
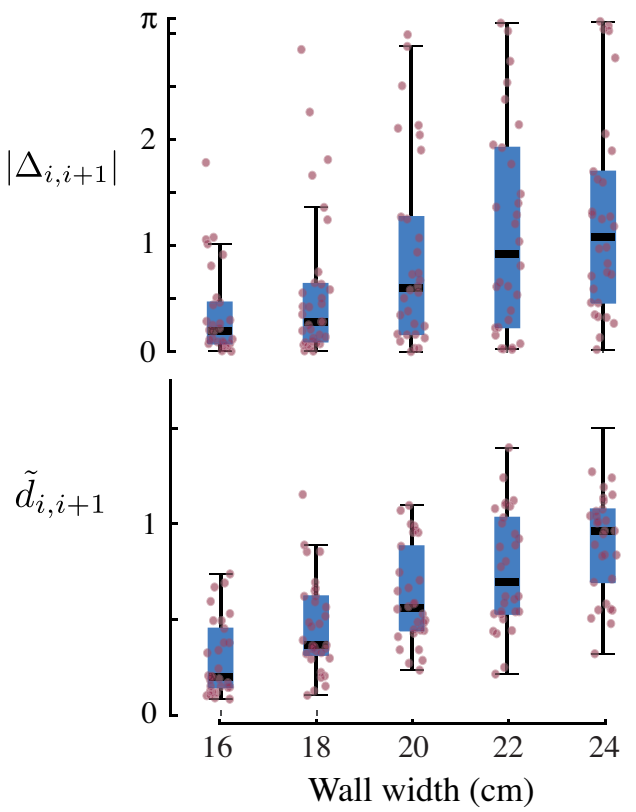

FIG. 8. Three-link robot experiments demonstrating synchronization through collisions. (a) Simple robot with three rigid links and two servo motors generating undulatory motion. (b) Motion of the joints when controlled as a phase oscillator shown for the experimental system (left) and a simulation (right). (c) Experiments with four robots in a controlled width channel demonstrating synchronization of movement over time. (d) Phase difference $\Delta_{i, i+1}$ and lateral separation $\tilde{d}_{i, i+1}$ between neighboring robots. (e) Phase difference and separation distance between robots after 15 periods of oscillation from five different wall-width experiments (10 trials each). Boxplots show 25\%-75\% confidence intervals (blue box) and the median (horizontal black line). All observations are plotted as red circles. 
velocity from the servos and thus incorporates proprioceptive feedback to generate autonomous oscillations, consistent with the direct-drive motors of the previous experiment.

We simulate the three-link robots using the Project Chrono multibody physics simulation engine [49]. In simulation, we directly control the torque of the rotational joints consistent with the previous two-motor experiment [Eq. (11)]. Contact interactions in the simulation are modeled through shortrange repulsive viscoelastic interactions, and we add stokesdrag fluid forces to the robot links according to the method in Ref. [50] to mimic the damping from friction in experiment. In both the experiment and simulation, we incorporate methods to enforce a constant phase difference of $\left(\frac{2}{3} \pi\right)$ between joints to produce traveling-wave body undulations. We slightly modify the actuation equation by adding a coupling term $(\lambda)$ between joints $\alpha_{1}, \alpha_{2}$,

$$
\tau_{i, j}=-k\left(x_{i, j}+\lambda_{j} x_{i, j}\right)+\left(c-\mu x_{i, j}^{2}\right) \dot{x}_{i, j},
$$

in which subscripts $i=1,2,3, \ldots$ represent the number of robots and $j=1,2$ represent the two joints of robot $i$. The coupling constants for the two joints are $\lambda_{1}=1.5$ and $\lambda_{2}=-0.5$, and the position $x_{i, \bar{j}}$ refers to the opposite joint of the robot. The position and torque control methods of the experiment and simulation produce body undulations of the robot with a constant frequency and phase difference [Fig. 8(b)]. In experiment, the frictional interactions between the robot links and ground cause perturbations to the robot joint motion; however, this does not affect the synchronization behavior of the robots.

To observe whether multiple undulatory robots will synchronize their gaits through contact, we put groups of four robots within a confined rectangular channel [Fig. 8(c); Video 2 in Ref. [40]]. In experiments, we only test configurations where the robots were aligned longitudinally, but we test the effect of longitudinal misalignment in simulation. The rectangular channel is $55 \mathrm{~cm}$ long, and we test five different widths, $w \in[16,18,20,22,24] \mathrm{cm}$ with 10 trials at each width. The experiment begins with the robots evenly spaced in the lateral direction and at random initial phases. After $30 \mathrm{~s}$ (approximately 15 periods of oscillation), we stop the experiment and measure the final phase difference $\Delta_{i, i+1}$ and spatial distance $\tilde{d}_{i, i+1}$ between neighboring robot pairs [Fig. 8(d)]. Increasing the wall width causes both $\Delta_{i, i+1}$ and $\tilde{d}_{i, i+1}$ to increase [Fig. 8(e)].

We perform similar three-link robot synchronization experiments in simulation. In addition to simulating the experiments performed with physical robots, we also increase the number of robots and the confinement arena size to represent two-dimensional simulations in which robots occupy a rectangular region. Qualitatively, the one-dimensional and two-dimensional arenas exhibit similar spatial and phase effects, with nearby robots influencing each other in the undulatory phase and reaching compatibility.
In all experiments $(n=50)$ and simulations $(n=210)$, the three-link robots adjust their undulatory phase through collisions, and the final states are well characterized by the theoretical model of Sec. II. When we examine the nearestneighbor phase difference versus lateral separation, we see that all robot-robot interactions lead to phase and distance states that are near or below the compatibility condition [Eq. (6); black line in Fig. 9]. Critically, we never observed antiphase synchronization as we did in the earlier two-joint experiments from Sec. III or the immobile lattice simulations from Sec. IV. The lack of antiphase behavior is understandable from the mobile simulations in Sec. IV; when robots collide, they push each other away, and this spatial repulsion drives them out of contact before they synchronize to the antiphase.

The extremely good agreement we observe from both the simulation and experiment with the compatibility model indicates that contact interactions have an important role in collective phase dynamics. Initial states outside of compatibility evolve to synchronized movement when spacing is small, and compatible phases evolve at larger spacing. It is important to note that the mobile robots in simulation and experiment can displace and rotate with respect to each other, thus indicating that the phase dynamics model of Sec. II is robust to misalignment and natural variation. However, it remains to be demonstrated what benefits gait synchronization would have for undulatory collectives. In the last section, we compare time-dependent actuation in an asynchronous group versus undulatory generation through autonomous oscillators, which enables synchronization.

\section{SYNCHRONIZATION MINIMIZES CONTACT FORCES IN UNDULATORY GROUPS}

In this last section, we seek to determine the potential benefit of gait synchronization for collectives. There are likely many metrics that could be influenced by synchronization: locomotion energetics and collective sensing, for example. Here, we focus on the interaction forces between robots that occur when in high-density spatial arrangements.

We conduct simulations with groups of ten three-link robots in a confined rectangular volume [Fig. 10(a)]. Initially, the robot joints are actuated through a timedependent position control signal with fixed frequency and amplitude

$$
\bar{\alpha}_{i, j}=A \sin \left(\phi_{i}+\frac{2 \pi}{3}(j-1)-2 \pi f t\right)
$$

in which subscripts $i=1,2,3, \ldots$ represent the number of robots and $j=1,2$ represent the two joints of robot $i$. The position command is converted to a control torque through a proportional control law $\tau_{i, j}=-k\left(\alpha_{i, j}-\bar{\alpha}_{i, j}\right)$, where $\alpha_{i, j}$ is the actual joint angle. The proportionality constant $k$ determines how much torque the actuators exert when the 

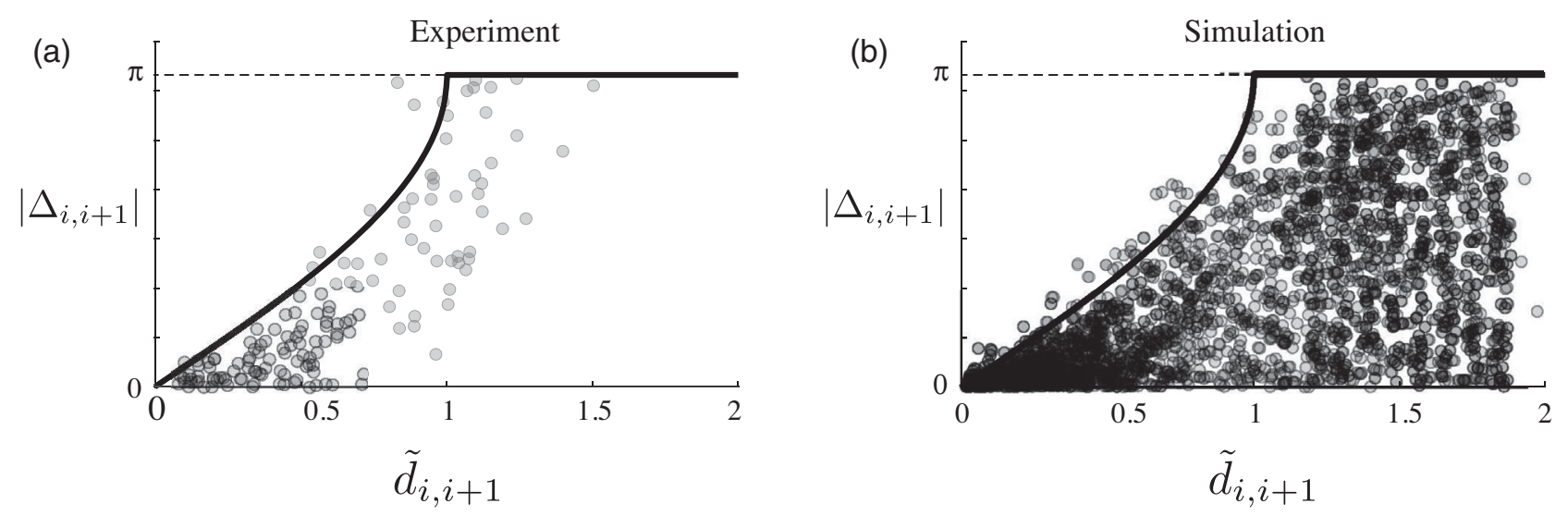

FIG. 9. Robots adjust their undulatory phase and lateral distance according to the theoretical model [black curve; Eq. (6)]. Results from simulation $(n=210)$ and experiments $(n=50)$ at random initial conditions are shown.

position deviates from the time-dependent sinusoidal commands and can be considered as a controller stiffness. We perform simulations across $k \in[0.015,0.15] \frac{\mathrm{Nm}}{\mathrm{rad}}$. For each control stiffness, we perform ten simulations at random initial phases $\left[\phi_{i} \in[-\pi, \pi]\right.$ in Eq. (13)]. The frequency $(0.8 \mathrm{~Hz})$ and amplitude $(0.8 \mathrm{rad})$ are chosen to match the oscillation kinematics when the robots are under limit-cycle control [Eq. (12)].

To enforce contact and collisions, we slowly move the top and bottom walls inwards towards the arena center. The rectangular region has a constant width of $0.6 \mathrm{~m}$, and at the beginning of the simulation, the lateral walls are $2.0 \mathrm{~m}$ apart. The width of the lateral walls is decreased at constant velocity from $10 \mathrm{~s}$ to $100 \mathrm{~s}$ while the robots oscillate, stopping at a lateral width of $0.3 \mathrm{~m}$ for the rest of the simulation [Fig. 10(a)]. The robots are controlled through time-dependent sinusoidal actuation during the first $200 \mathrm{~s}$ and switched to phase-oscillator control from 200-300 s.
We record the oscillatory phase and the contact forces acting on all robot links during each time step. The mean force between robots under the phase-fixed control is significantly larger than that under the phase-oscillator control mode in which synchronization occurs [Fig. 10(b)]. The interaction forces between the robots in the highdensity environment are large because the oscillation phases are incompatible, resulting in collisions. These collisions persist and repeat because the phase differences are fixed, leading to a fluctuating mean force with constant time-averaged behavior [Fig. 10(b)]. However, when robots are switched to the phase-oscillator control mode, the collisions between robots quickly drive the robot group to synchronization. The median contact force is less than $0.1 \mathrm{~N}$ during the phase-oscillator control mode, indicating a large reduction in contact forces.

This section demonstrates that robots with undulatory phase differences can experience large contact forces as (a)

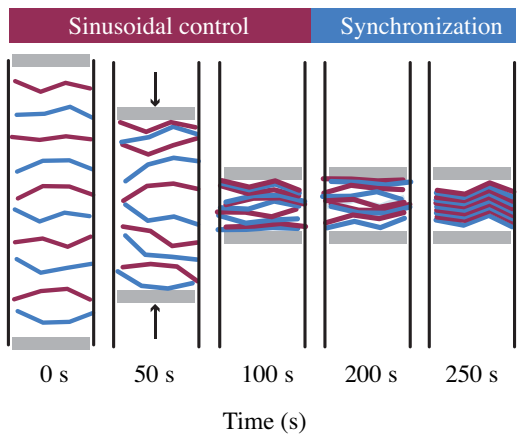

(b)

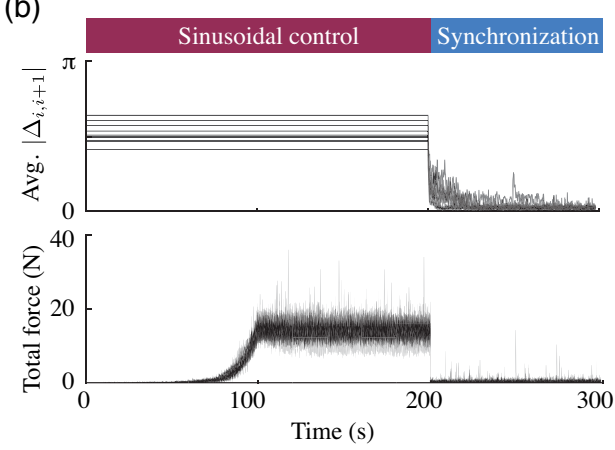

(c)

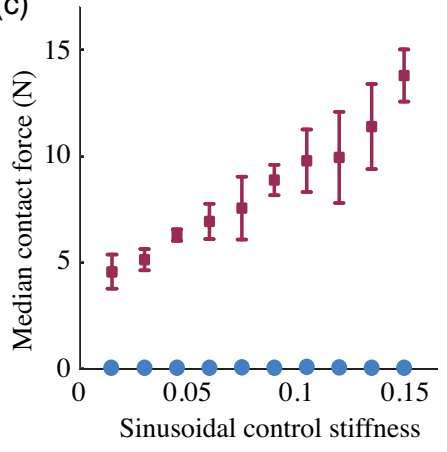

FIG. 10. Decrease of interaction forces in undulatory groups when the robots' gaits synchronize. (a) Ten robots in a rectangular arena oscillating through time-dependent sinusoidal control. The top and bottom walls are slowly brought together, resulting in persistent collisions. After $200 \mathrm{~s}$, joint control is switched from time-dependent to phase-oscillator actuation, and the robots synchronize. (b) Phase difference and contact forces plotted versus time. During time-dependent sinusoidal control, the robot phase differences are maintained, and contact forces are large. When phase-oscillator control is switched on, the robots synchronize, causing the phase difference and contact forces to decrease. Ten replicate experiments are overlaid, and force is filtered with a running average of $0.1 \mathrm{~s}$. (c) Median contact forces during a steady state for time-dependent sinusoidal control (purple squares, calculated from 100-200 s) and limit-cycle control (blue circles, calculated from 200-300 s). The forces during time-dependent sinusoidal control linearly increase with the proportional control constant (the effective "stiffness"). 
they push against each other. However, when synchronized to the same undulatory phase, the collisions reduce to a small and negligible magnitude. Contact forces between robots can be a significant problem and lead to rapid wear and failure. Similar negative consequences are likely to occur in biological collectives where repeated high-force contact can lead to higher energy expenditure and potential injury.

\section{DISCUSSION}

Our results have demonstrated that inelastic collisions between undulatory robots can produce novel phase dynamics such as in-phase and antiphase synchronization, and compatible oscillations that persist without contact. The behavior of larger robot groups tends towards phase compatibility, and once achieved, the group is effectively decoupled because collisions will no longer occur unless perturbed. The compatible state is similar to the "cohesive" state originally introduced for the Kuramoto system [51] in which cohesive oscillators remain within a bounded phase difference for all time. Compatibility is a beneficial property for undulatory groups because it minimizes the contact forces between individuals and thus likely reduces energetics, fatigue, and damage. Critically, this beneficial collective behavior emerges naturally from the physics of inelastic contact and simply requires that undulatory motion be generated through an autonomous oscillator so that phases between robots can "slip" through interactions. In additional simulations and experiments, we have demonstrated that this behavior is insensitive to the particular control law that generates undulation.

The coefficient of restitution from inelastic collisions between robots is the lone governing parameter for phase dynamics among these contact-coupled groups. Inelastic contact interactions generate a wide array of collective behaviors in driven or active nonlinear systems, such as pattern formation [52,53], particle aggregation [29,54], and swarming $[55,56]$. However, the ability of repulsive contact interactions to drive attractive phase dynamics in oscillators has not been observed. Our phase model is able to explain how phase attraction and repulsion emerge from inelastic collisions. When undulatory systems are in close proximity, the collisional interactions between their limit cycles drive their phase difference to be smaller. However, when the separation distance is large, collisions drive the phase difference to grow and generate a stable antiphase mode. Extending these interactions to an oscillator lattice, we have shown that phase repulsion can destroy long-range order when the oscillator base is immobilized, while mobile undulatory systems always reach compatibility.

Our inspiration for this study comes from collective movement in worm groups in which body and appendage oscillations may occur in close proximity. Recent work has demonstrated that collisional interactions in arrays of cilia can generate synchronization, metachronal wave propagation, and jammed states, dependent on separation [38].
Similarly, recent observations of small worms that swim by laterally oscillating their bodies have illustrated that groups of worms tend to synchronize their oscillatory phase when in close proximity $[16,57,58]$. Genetic manipulations of these worms illustrated that external sensory responses (exteroception) were not necessary for synchronization, and instead, the authors argued that collisional ("steric") interactions could produce synchronization [16]. Our results provide a potential explanation for the observed gait synchronization: Body oscillations that are governed by internal proprioceptive neural feedback can exhibit emergent synchronization through collisional body interactions alone.

The system explored in this experiment has appreciable inertial dynamics and momentum transfer through collision. However, in the systems we take inspiration from, such as small oscillatory organisms in fluids, inertial dynamics are likely not relevant. Thus, it is important to consider how these results may apply across inertial and noninertial active matter systems. We propose that contactcoupled oscillators in both the inertial and noninertial regimes are captured by the coefficient of restitution in our phase model. When $r=0$, the oscillators do not rebound but instead "stick" together, which models the noninertial behavior of oscillators such as cilia and worms in overdamped viscous environments. However, for $r>0$, systems exhibit significant rebounding as they collide, which captures the behavior of inertial oscillatory systems and can lead to antiphase synchronization [Fig. 3(b)]. The reduction of contact-coupled oscillators to a simple model in which $r$ is the only governing parameter allows us to explore these systems across inertial to noninertial regimes. This will be of interest in future studies and in comparisons between model predictions and observations from active matter and swarm robot systems in experiment.

This work has relevance to the fields of swarm and collective robotics where a critical goal is to design distributed control laws that lead to desired, beneficial, emergent behaviors of the group [59]. Recent work in swarm robotics has embraced contact and collisional interactions as a means of coordinating robot group behaviors [60-68], and other recent work has leveraged collisions [69-72] for maneuvering individual robots. Our work demonstrates that designing appropriate limit cycles to actuate the rhythmic motion of robots can lead to emergent synchronization and drastically reduce the contact forces. Thus, the desired collective behaviors of the group are encoded within the gait generation algorithm of the individuals. Future capabilities can thus build on to the collective behaviors studied here. For example, subsets of informed individuals could "herd" the group through contact interactions controlling collective motion.

The coupling of oscillatory dynamics with mobility is an exciting future direction for active matter systems such as biological or robotics swarms. Previous work on mobile phase oscillators in which the phase differences can influence motion of the mobile systems has demonstrated novel collective flocking and pattern formation behaviors [73-75]. 
However, there has been little work to consider how the mechanical collisions between oscillating moving individuals drive collective synchrony or motion patterns. In recent experiments, three-link "smarticle" robots have demonstrated how stochastic interactions among neighboring oscillating robots can drive emergent and controlled collective behavior [37]. However, currently, smarticle systems do not have oscillatory phase dynamics, and thus synchronization has not been explored. In our work, the oscillator phase is intrinsically tied to the undulatory motion of the robotic joint. Thus, phase and motion are explicitly coupled. Future swarm systems that take advantage of the phase dynamics from inelastic collisions may enable emergent synchronization of mobile undulatory robots purely through contact, thus simplifying swarm robot motion control.

\section{ACKNOWLEDGMENTS}

We acknowledge helpful discussions with Dan Goldman and Paul Umbanhowar. We thank the UCSD Department of Mechanical \& Aerospace Engineering for funding support. This material is based upon work supported by the Office of Naval Research under Grant No. N00014-20-1-2373 and the National Science Foundation under Grant No. 1935324.

\section{APPENDIX A: DERIVATION OF CONTACT MAP}

In this section, we derive the collision-to-collision phase map presented in Sec. II. To derive this map, we have to first consider how to represent the precollision phases $\phi_{i}^{-}$in terms of only the phase difference $\Delta=\phi_{2}-\phi_{1}$. We seek to solve for the collision phases using only the phase difference between oscillators, $\Delta$. We begin by introducing an intermediate variable $\kappa$ such that

$$
\begin{aligned}
& \phi_{1}=\kappa-\frac{\Delta}{2}, \\
& \phi_{2}=\kappa+\frac{\Delta}{2} .
\end{aligned}
$$

The collision condition [Eq. (1) in main text] is

$$
2 \tilde{d}=\cos \left(\phi_{2}\right)-\cos \left(\phi_{1}\right),
$$

and we expand this into the form

$$
\begin{gathered}
2 \tilde{d}=\cos \left(\phi_{1}\right)-\cos \left(\phi_{2}\right) \\
=\cos \left(\kappa-\frac{\Delta}{2}\right)-\cos \left(\kappa+\frac{\Delta}{2}\right) \\
=-2 \sin (\kappa) \sin \left(-\frac{\Delta}{2}\right),
\end{gathered}
$$

which yields the relationship

$$
\kappa=\arcsin \left(\tilde{d} \csc \left(\frac{\Delta}{2}\right)\right) .
$$

This equation allows us to determine, for a given initial $\Delta$, what the individual phases of the oscillators are at collision by substituting $\kappa$ into Eqs. (A1) and (A2).

Our goal here is to solve for the return map between collisions as a function of $\Delta$. To do this, we take the following steps: (1) Solve for $\phi_{1}$ and $\phi_{2}$ at collision from Eqs. (A1), (A2), and (A8); (2) apply the velocity update rule for inelastic collisions from Eqs. (2) and (3); (3) determine the postcollision phases for the oscillators from Eq. (4). Since $\omega$ is the same between each oscillator and they evolve independently until colliding, the postcollision phase difference $\Delta^{(n,+)}$ is exactly the same phase difference of the next collision, $\Delta^{(n+1,-)}$. We have introduced the superscript notation where the first value indexes the collision, and the \pm denotes whether the value is before $(-)$ or after $(+)$ the indexed collision:

$$
\begin{gathered}
\Delta^{(n,+)}=\phi_{2}^{(n,+)}-\phi_{1}^{(n,+)} \\
=\operatorname{atan}\left[-\frac{\dot{x}_{2}^{(n,+)}}{x_{2}^{(n,+)}}\right]-\operatorname{atan}\left[-\frac{\dot{x}_{1}^{(n,+)}}{\left.x_{1}^{(n,+)}\right]}\right. \\
=\operatorname{atan}\left[\frac{(1-r) \dot{x}_{1}^{(n,-)}+(1+r) \dot{x}_{2}^{(n,-)}}{2 x_{1}^{(n,-)}}\right]-\operatorname{atan}\left[\frac{(1-r) \dot{x}_{2}^{(n,-)}+(1+r) \dot{x}_{1}^{(n,-)}}{2 x_{2}^{(n,-)}}\right] \\
=\operatorname{atan}\left[\frac{\dot{x}_{1}^{(n,-)}+\dot{x}_{2}^{(n,-)}-r\left(\dot{x}_{1}^{(n,-)}-\dot{x}_{2}^{(n,-)}\right)}{2 x_{1}^{(n,-)}}\right]-\operatorname{atan}\left[\frac{\dot{x}_{1}^{(n,-)}+\dot{x}_{2}^{(n,-)}+r\left(\dot{x}_{1}^{(n,-)}-\dot{x}_{2}^{(n,-)}\right)}{2 x_{2}^{(n,-)}}\right] \\
=\operatorname{atan}\left[\frac{(1-r) \sin \left(\kappa+\frac{\Delta^{(n,-)}}{2}\right)+(1+r) \sin \left(\kappa-\frac{\left.\Delta^{(n,-)}\right)}{2}\right)}{2 \cos \left(\kappa+\frac{\Delta^{(n,-)}}{2}\right)}\right]-\operatorname{atan}\left[\frac{(1-r) \sin \left(\kappa-\frac{\Delta^{(n,-)}}{2}\right)+(1+r) \sin \left(\kappa+\frac{\Delta^{(n,-)}}{2}\right)}{2 \cos \left(\kappa-\frac{\Delta^{(n,-)}}{2}\right)}\right]
\end{gathered}
$$




$$
=\operatorname{atan}\left[\frac{\sin (\kappa) \cos \left(\frac{\Delta^{(n,-)}}{2}\right)-r \cos (\kappa) \sin \left(\frac{\Delta^{(n,-)}}{2}\right)}{\cos \left(\kappa+\frac{\Delta^{(n,-)}}{2}\right)}\right]-\operatorname{atan}\left[\frac{\sin (\kappa) \cos \left(\frac{\Delta^{(n,-)}}{2}\right)+r \cos (\kappa) \sin \left(\frac{\Delta^{(n,-)}}{2}\right)}{\cos \left(\kappa-\frac{\Delta^{(n,-)}}{2}\right)}\right] .
$$

Since $\Delta^{(n,+)}=\Delta^{(n+1,-)}$, we have derived the mapping from the collision phase immediately after the $n$ collision to the phase immediately after the $n+1$ collision. Thus, we can drop the \pm superscripts, and we arrive at the final collision-tocollision return map,

$$
\Delta^{(n+1)}=\operatorname{atan}\left[\frac{\sin (\kappa) \cos \left(\frac{\Delta^{(n)}}{2}\right)-r \cos (\kappa) \sin \left(\frac{\Delta^{(n)}}{2}\right)}{\cos (\kappa) \cos \left(\frac{\Delta^{(n)}}{2}\right)-\sin (\kappa) \sin \left(\frac{\Delta^{(n)}}{2}\right)}\right]-\operatorname{atan}\left[\frac{\sin (\kappa) \cos \left(\frac{\Delta^{(n)}}{2}\right)+r \cos (\kappa) \sin \left(\frac{\Delta^{(n)}}{2}\right)}{\cos (\kappa) \cos \left(\frac{\Delta^{(n)}}{2}\right)+\sin (\kappa) \sin \left(\frac{\Delta^{(n)}}{2}\right)}\right] .
$$

\section{APPENDIX B: EXPERIMENT DETAILS}

\section{Motor control and limit-cycle generation}

Each motor was controlled by an ODrive brushless dc motor controller (ODrive Robotics). The ODrive provides closed-loop current control for each motor, and we set the maximum current limit to $30 \mathrm{~A}$. The motor current control was performed on a computer in Python. At every update loop, the motor current was computed using the following equation:

$$
i=-k \theta+c \dot{\theta}-\mu \theta^{2} \dot{\theta}+\beta \operatorname{sgn}(\theta),
$$

with the following parameters:

\begin{tabular}{lll}
\hline Variable & Motor 0 & Motor 1 \\
\hline$k$ & $3.9 \mathrm{~A} / \mathrm{rad}$ & $3.3 \mathrm{~A} / \mathrm{rad}$ \\
$\mu$ & $0.24 \mathrm{~A} \mathrm{~s} / \mathrm{rad}^{3}$ & $0.24 \mathrm{~A} \mathrm{~s} / \mathrm{rad}^{3}$ \\
$c$ & $0.009 \mathrm{~A} \mathrm{~s} / \mathrm{rad}^{2}$ & $0.009 \mathrm{~A} \mathrm{~s} / \mathrm{rad}$ \\
$\beta$ & $0.25 \mathrm{~A}$ & $0.25 \mathrm{~A}$ \\
\hline
\end{tabular}

The constants were selected so that each motor exhibited limit-cycle oscillations of approximately sinusoidal motion with equal amplitude $\left(A_{1}=44.4 \pm 0.9\right.$ degrees and $A_{2}=$ $44.3 \pm 1.6$ degrees $)$ and equal frequency $\left(\omega_{1}=2.61 \pm\right.$ $0.04 \mathrm{~Hz}$ and $\omega_{2}=2.63 \pm 0.03 \mathrm{~Hz}$ ). The $\beta$ term in the motor control equation helped overcome the frictional resistance of the motor bearings. Without this term, the motor dynamics exhibited a stable fixed point at $(\theta, \dot{\theta})=$ $(0,0)$ with a small region of attraction around this point.

\section{Collision dynamics}

A rigid robot link of length $9 \mathrm{~cm}$ was attached to each motor. The link was waterjet cut from 9.5-mm-thick aluminum and rigidly fastened to the motor. A 3D printed adapter was attached to the end of each link, which provided an impact surface for the two links to interact with each other (Fig. 11). The colliding surface was an elastic sphere, a bouncy ball, purchased from a commercial vendor.
To determine the coefficient of restitution of the impacting surfaces, we performed a series of experiments. The links were accelerated towards each other at a constant motor current (selected at random between 0 and $2 \mathrm{~A}$ ) for $200 \mathrm{~ms}$, after which the current was set to 0 and the motors and links glided towards each other, impacting and rebounding. We measured the motor speed immediately prior to the collision and immediately after the collision and computed the coefficient of restitution using the equation $\dot{x}_{1}^{+}-\dot{x}_{2}^{+}=-r\left(\dot{x}_{1}^{-}-\dot{x}_{2}^{-}\right)$. We found a coefficient of restitution of $r=0.67 \pm 0.02$.

\section{Simulation}

We performed numerical simulations of colliding oscillator pairs and collectives. Simulations were performed in both Matlab and C++ using the library "odeint" and a variable time-step integrator with absolute and relative tolerances of $1 \times 10^{-6}$. An event detection scheme was used in both simulation environments to detect oscillator collisions. At each collision, the numerical integration was halted, the inelastic collision model was implemented, and the integration was reinitialized with the new postcollision state. In the simulations with more than two oscillators, simultaneous collisions between more than one oscillator pair were not observed. (a)

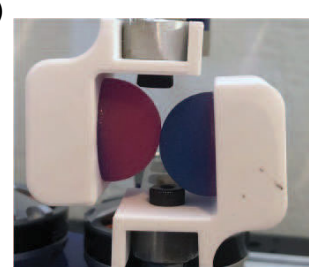

(b)

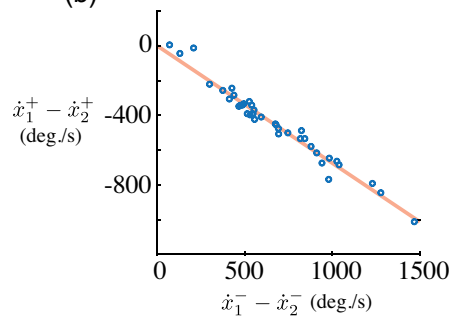

FIG. 11. Measurement of coefficient of restitution for experiment. (a) Impacting surfaces. (b) Coefficient of restitution measurement. The equation is given in the text. 
[1] A. T. Winfree, The Geometry of Biological Time (Springer, New York, 2001).

[2] A. T. Winfree, Biological Rhythms and the Behavior of Populations of Coupled Oscillators, J. Theor. Biol. 16, 15 (1967).

[3] K. Y. Wan and R. E. Goldstein, Coordinated Beating of Algal Flagella is Mediated by Basal Coupling, Proc. Natl. Acad. Sci. U.S.A. 113, E2784 (2016).

[4] D. R. Brumley, K. Y. Wan, M. Polin, and R. E. Goldstein, Flagellar Synchronization through Direct Hydrodynamic Interactions, eLife 3, e02750 (2014).

[5] V. F. Geyer, F. Jülicher, J. Howard, and B. M. Friedrich, Cell-Body Rocking Is a Dominant Mechanism for Flagellar Synchronization in a Swimming Alga, Proc. Natl. Acad. Sci. U.S.A. 110, 18058 (2013).

[6] D. R. Brumley, M. Polin, T. J. Pedley, and R. E. Goldstein, Hydrodynamic Synchronization and Metachronal Waves on the Surface of the Colonial Alga Volvox Carteri, Phys. Rev. Lett. 109, 268102 (2012).

[7] J. Han and C. S. Peskin, Spontaneous Oscillation and FluidStructure Interaction of Cilia, Proc. Natl. Acad. Sci. U.S.A. 115, 4417 (2018).

[8] W. Gilpin, M.S. Bull, and M. Prakash, The Multiscale Physics of Cilia and Flagella, Nat. Rev. Phys. 2, 74 (2020).

[9] J. W. Newbolt, J. Zhang, and L. Ristroph, Flow Interactions between Uncoordinated Flapping Swimmers Give Rise to Group Cohesion, Proc. Natl. Acad. Sci. U.S.A. 116, 2419 (2019).

[10] A. U. Oza, L. Ristroph, and M. J. Shelley, Lattices of Hydrodynamically Interacting Flapping Swimmers, Phys. Rev. X 9, 041024 (2019).

[11] A. D. Becker, H. Masoud, J. W. Newbolt, M. Shelley, and L. Ristroph, Hydrodynamic Schooling of Flapping Swimmers, Nat. Commun. 6, 8514 (2015).

[12] N. Gravish, G. Gold, A. Zangwill, M. A. D. Goodisman, and D. I. Goldman, Glass-like Dynamics in Confined and Congested Ant Traffic, Soft Matter 11, 6552 (2015).

[13] J. Aguilar, D. Monaenkova, V. Linevich, W. Savoie, B. Dutta, H.-S. Kuan, M. D. Betterton, M. A. D. Goodisman, and D. I. Goldman, Collective Clog Control: Optimizing Traffic Flow in Confined Biological and Robophysical Excavation, Science 361, 672 (2018).

[14] J. Buhl, D. J. T. Sumpter, I. D. Couzin, J. J. Hale, E. Despland, E. R. Miller, and S. J. Simpson, From Disorder to Order in Marching Locusts, Science 312, 1402 (2006).

[15] Z. Csahok and T. Vicsek, Traffic Models with Disorder, J. Phys. A 27, L591 (1994).

[16] J. Yuan, D. M. Raizen, and H. H. Bau, Gait Synchronization in Caenorhabditis Elegans, Proc. Natl. Acad. Sci. U.S.A. 111, 6865 (2014).

[17] T. Danino, O. Mondragón-Palomino, L. Tsimring, and J. Hasty, A Synchronized Quorum of Genetic Clocks, Nature (London) 463, 326 (2010).

[18] A. M. Tayar, E. Karzbrun, V. Noireaux, and R. H. Bar-Ziv, Synchrony and Pattern Formation of Coupled Genetic Oscillators on a Chip of Artificial Cells, Proc. Natl. Acad. Sci. U.S.A. 114, 11609 (2017).

[19] R. E. Mirollo and S. H. Strogatz, Synchronization of PulseCoupled Biological Oscillators, SIAM J. Appl. Math. 50, 1645 (1990).
[20] R. Sarfati, J. C. Hayes, É. Sarfati, and O. Peleg, Spatiotemporal Reconstruction of Emergent Flash Synchronization in Firefly Swarms via Stereoscopic 360-Degree Cameras, J. R. Soc. Interface 17, 20200179 (2020).

[21] S. H. Strogatz, Nonlinear Dynamics and Chaos: With Applications to Physics, Biology, Chemistry and Engineering (Westview Press, Boulder, CO, 2000).

[22] A. Pikovsky, J. Kurths, M. Rosenblum, and J. Kurths, Synchronization: A Universal Concept in Nonlinear Sciences (Cambridge University Press, Cambridge, England, 2003).

[23] M. Bennett, M. F. Schatz, H. Rockwood, and K. Wiesenfeld, Huygens's Clocks, Proc. R. Soc. A 458, 563 (2002).

[24] E. Marder and D. Bucher, Central Pattern Generators and the Control of Rhythmic Movements, Curr. Biol. 11, R986 (2001).

[25] A. J. Ijspeert, Central Pattern Generators for Locomotion Control in Animals and Robots: A Review, Neural Netw. 21, 642 (2008).

[26] K. Matsuoka, Sustained Oscillations Generated by Mutually Inhibiting Neurons with Adaptation, Biol. Cybern. 52, 367 (1985).

[27] N. Kopell and G. B. Ermentrout, Coupled Oscillators and the Design of Central Pattern Generators, Math. Biosci. 90, 87 (1988).

[28] N. Topic and T. Pöschel, Inelastic Collapse of Perfectly Inelastic Particles, Commun. Phys. 2, 85 (2019).

[29] S. McNamara and W. R. Young, Inelastic Collapse in Two Dimensions, Phys. Rev. E 50, R28 (1994).

[30] R. P. Behringer and B. Chakraborty, The Physics of Jamming for Granular Materials: A Review, Rep. Prog. Phys. 82, 012601 (2019).

[31] H. M. Jaeger, S. R. Nagel, and R. P. Behringer, Granular Solids, Liquids, and Gases, Rev. Mod. Phys. 68, 1259 (1996).

[32] S. Ramaswamy, The Mechanics and Statistics of Active Matter, Annu. Rev. Condens. Matter Phys. 1, 323 (2010).

[33] M. R. Shaebani, A. Wysocki, R. G. Winkler, G. Gompper, and H. Rieger, Computational Models for Active Matter, Nat. Rev. Phys. 2, 181 (2020).

[34] Y. Yang, J. Elgeti, and G. Gompper, Cooperation of Sperm in Two Dimensions: Synchronization, Attraction, and Aggregation through Hydrodynamic Interactions, Phys. Rev. E 78, 061903 (2008).

[35] Y. Yang, V. Marceau, and G. Gompper, Swarm Behavior of Self-Propelled Rods and Swimming Flagella, Phys. Rev. E 82, 031904 (2010).

[36] P. Chvykov, T. A. Berrueta, A. Vardhan, W. Savoie, A. Samland, T. D. Murphey, K. Wiesenfeld, D. I. Goldman, and J. L. England, Low Rattling: A Predictive Principle for Self-Organization in Active Collectives, Science 371, 90 (2021).

[37] W. Savoie, T. A. Berrueta, Z. Jackson, A. Pervan, R. Warkentin, S. Li, T. D. Murphey, K. Wiesenfeld, and D. I. Goldman, A Robot Made of Robots: Emergent Transport and Control of a Smarticle Ensemble, Sci. Robotics 4 (2019).

[38] R. Chelakkot, M. F. Hagan, and A. Gopinath, Synchronized Oscillations, Traveling Waves, and Jammed Clusters Induced by Steric Interactions in Active Filament Arrays, Soft Matter 17, 1091 (2020). 
[39] J. Buchli, L. Righetti, and A. J. Ijspeert, Engineering Entrainment and Adaptation in Limit Cycle Systems: From Biological Inspiration to Applications in Robotics, Biol. Cybern. 95, 645 (2006).

[40] See Supplemental Material at http://link.aps.org/ supplemental/10.1103/PhysRevX.11.031051 for Supplemental Videos 1-3 showing robot experiments.

[41] L. S. Tsimring, N. F. Rulkov, M. L. Larsen, and M. Gabbay, Repulsive Synchronization in an Array of Phase Oscillators, Phys. Rev. Lett. 95, 014101 (2005).

[42] E. M. Purcell, Life at Low Reynolds Number, Am. J. Phys. 45, 3 (1977).

[43] J. E. Avron and O. Raz, A Geometric Theory of Swimming: Purcell's Swimmer and Its Symmetrized Cousin, New J. Phys. 10, 063016 (2008).

[44] D. Tam and A. E. Hosoi, Optimal Stroke Patterns for Purcell's Three-Link Swimmer, Phys. Rev. Lett. 98, 068105 (2007).

[45] S. Alben, Efficient Sliding Locomotion of Three-Link Bodies, Phys. Rev. E 103, 042414 (2021).

[46] F. Jing and S. Alben, Optimization of Two- and Three-Link Snakelike Locomotion, Phys. Rev. E 87, 022711 (2013).

[47] R. L. Hatton, Y. Ding, H. Choset, and D. I. Goldman, Geometric Visualization of Self-Propulsion in a Complex Medium, Phys. Rev. Lett. 110, 078101 (2013).

[48] Y. Ozkan-Aydin, D. I. Goldman, and M. S. Bhamla, Collective Dynamics in Entangled Worm and Robot Blobs, Proc. Natl. Acad. Sci. U.S.A. 118, e2010542118 (2021).

[49] A. Tasora, R. Serban, H. Mazhar, A. Pazouki, D. Melanz, J. Fleischmann, M. Taylor, H. Sugiyama, and D. Negrut, Chrono: An Open Source Multi-physics Dynamics Engine, in High Performance Computing in Science and Engineering (Springer International Publishing, Cham, 2016), pp. 19-49, 10.1007/978-3-31940361-8_2.

[50] R. L. Hatton and H. Choset, Geometric Swimming at Low and High Reynolds Numbers, IEEE Trans. Rob. 29, 615 (2013).

[51] F. Dörfler and F. Bullo, On the Critical Coupling for Kuramoto Oscillators, SIAM J. Appl. Dyn. Syst. 10, 1070 (2011).

[52] I. S. Aranson and L.S. Tsimring, Pattern Formation of Microtubules and Motors: Inelastic Interaction of Polar Rods, Phys. Rev. E 71, 050901(R) (2005).

[53] T. Shinbrot, Competition between Randomizing Impacts and Inelastic Collisions in Granular Pattern Formation, Nature (London) 389, 574 (1997).

[54] D. Goldman, M. D. Shattuck, C. Bizon, W. D. McCormick, J. B. Swift, and H. L. Swinney, Absence of Inelastic Collapse in a Realistic Three Ball Model, Phys. Rev. E 57, 4831 (1998).

[55] D. Grossman, I. S. Aranson, and E. B. Jacob, Emergence of Agent Swarm Migration and Vortex Formation through Inelastic Collisions, New J. Phys. 10, 023036 (2008).

[56] A. Kudrolli, G. Lumay, D. Volfson, and L. S. Tsimring, Swarming and Swirling in Self-Propelled Polar Granular Rods, Phys. Rev. Lett. 100, 058001 (2008).

[57] A. Peshkov, S. McGaffigan, and A.C. Quillen, Wiggling Droplets: Metachronal Waves in Populations of Turbatrix Aceti, arXiv:2104.10316.
[58] A. C. Quillen, A. Peshkov, E. Wright, and S. McGaffigan, Metachronal Waves in Concentrations of Swimming Turbatrix Aceti Nematodes and an Oscillator Chain Model for Their Coordinated Motions, Phys. Rev. E 104, 014412 (2021).

[59] G.-Z. Yang, J. Bellingham, P. E. Dupont, P. Fischer, L. Floridi, R. Full, N. Jacobstein, V. Kumar, M. McNutt, R. Merrifield, B. J. Nelson, B. Scassellati, M. Taddeo, R. Taylor, M. Veloso, Z. L. Wang, and R. Wood, The Grand Challenges of Science Robotics, Sci. Robotics 3, eaar7650 (2018).

[60] F. Mondada, L. M. Gambardella, D. Floreano, S. Nolfi, J.-L. Deneuborg, and M. Dorigo, The Cooperation of SwarmBots: Physical Interactions in Collective Robotics, IEEE Robot. Autom. Mag. 12, 21 (2005).

[61] M. P. Nemitz, E. Olson, and A. A. Stokes, HoverBots: Embracing and Detecting Collisions Using Robots Designed for Manufacturability, in ICRA 2018 Workshop: Swarms: From Biology to Robotics and Back (2018).

[62] S. Mayya, P. Pierpaoli, G. Nair, and M. Egerstedt, Localization in Densely Packed Swarms Using Interrobot Collisions as a Sensing Modality, IEEE Trans. Rob. 35, 21 (2019).

[63] S. Mayya, P. Pierpaoli, G. Nair, and M. Egerstedt, Collisions as Information Sources in Densely Packed Multi-Robot Systems under Mean-Field Approximations, in Proceedings of Robotics: Science and Systems (2017), Vol. 13, 10.15607/ RSS.2017.XIII.044.

[64] S. Mayya, S. Wilson, and M. Egerstedt, Closed-Loop Task Allocation in Robot Swarms Using Inter-Robot Encounters, Swarm Intell. 13, 115 (2019).

[65] S. Mayya, G. Notomista, D. Shell, S. Hutchinson, and M. Egerstedt, Non-Uniform Robot Densities in Vibration Driven Swarms Using Phase Separation Theory, in 2019 IEEE/RSJ International Conference on Intelligent Robots and Systems (IROS) (2019), pp. 4106-4112, 10.1109/ IROS40897.2019.8967985.

[66] T. Schmickl, R. Thenius, C. Moeslinger, G. Radspieler, S. Kernbach, M. Szymanski, and K. Crailsheim, Get in Touch: Cooperative Decision Making Based on Robot-toRobot Collisions, Auton. Agent. Multi. Agent. Syst. 18, 133 (2009).

[67] C. Scholz, M. Engel, and T. Pöschel, Rotating Robots Move Collectively and Self-Organize, Nat. Commun. 9, 931 (2018).

[68] M. A. Karimi, V. Alizadehyazdi, B. Busque, H. M. Jaeger, and M. Spenko, A Boundary-Constrained Swarm Robot with Granular Jamming, in 2020 3rd IEEE International Conference on Soft Robotics (RoboSoft) (2020), pp. 291296, 10.1109/RoboSoft48309.2020.9115996.

[69] Y. Mulgaonkar, A. Makineni, L. Guerrero-Bonilla, and V. Kumar, Robust Aerial Robot Swarms without Collision Avoidance, IEEE Robot. Autom. Lett. 3, 596 (2018).

[70] Z. Lu, Z. Liu, G. J. Correa, and K. Karydis, Motion Planning for Collision-Resilient Mobile Robots in Obstacle-Cluttered Unknown Environments with Risk Reward Trade-offs, arXiv:2009.01973.

[71] M. Mote, M. Egerstedt, E. Feron, A. Bylard, and M. Pavone, Collision-Inclusive Trajectory Optimization for Free-Flying Spacecraft, J. Guid. Control Dyn. 43, 1247 (2020). 
[72] J. Zha and M.W. Mueller, Exploiting Collisions for Sampling-Based Multicopter Motion Planning, arXiv:2011 .04091 .

[73] K. P. O'Keeffe, H. Hong, and S. H. Strogatz, Oscillators that Sync and Swarm, Nat. Commun. 8, 1504 (2017).
[74] K. Uriu, S. Ares, A. C. Oates, and L. G. Morelli, Dynamics of Mobile Coupled Phase Oscillators, Phys. Rev. E 87, 032911 (2013).

[75] M. Frasca, A. Buscarino, A. Rizzo, L. Fortuna, and S. Boccaletti, Synchronization of Moving Chaotic Agents, Phys. Rev. Lett. 100, 044102 (2008). 